\title{
Virtual beams and the Klein paradox for the Klein-Gordon equation
}

\author{
A. Molgado, O. Morales and J.A. Vallejo \\ Facultad de Ciencias, Universidad Autónoma de San Luis Potosí*, \\ and Dual CP Institute of High Energy, México, \\ *Lat. Av. Salvador Nava s/n. Col. Lomas, 78290, San Luis Potosí, México. \\ e-mail:molgado@fc.uaslp.mx; jvallejo@fc.uaslp.mx
}

Received 2 February 2016; accepted 24 June 2016

\begin{abstract}
Whenever we consider any relativistic quantum wave equation we are confronted with the Klein paradox, which asserts that incident particles will suffer a surplus of reflection when dispersed by a discontinuous potential. Following recent results on the Dirac equation, we propose a solution to this paradox for the Klein-Gordon case by introducing virtual beams in a natural well-posed generalization of the method of images in the theory of partial differential equations. Thus, our solution considers a global reflection coefficient obtained from the two contributions, the reflected particles plus the incident virtual particles. Despite its simplicity, this method allows a reasonable understanding of the paradox within the context of the quantum relativistic theory of particles (according to the original setup for the Klein paradox) and without resorting to any quantum field theoretic issues.
\end{abstract}

Keywords: Klein-Gordon equation; Klein paradox; Method of images.

PACS: 03.65.Pm; 02.30.Jr.

\section{Introduction}

Back in the early days of quantum mechanics, following a suggestion of Debye, Schrödinger tried to find an equation describing the behavior of the waves introduced by De Broglie. His first attempt started with Einstein's equation for the relativistic energy

$$
E^{2}=p^{2} c^{2}+m^{2} c^{4},
$$

which he expected to be satisfied by the quantum waves. To this end, he observed that a possible solution for the wave equation (deduced from Maxwell's equations for the components of the electromagnetic field)

$$
\frac{\partial^{2} \Phi}{\partial x^{2}}-\frac{1}{c^{2}} \frac{\partial^{2} \Phi}{\partial t^{2}}=0
$$

is a plane monochromatic wave

$$
\Phi(x, t)=\Phi_{0} e^{i(k x-w t)},
$$

where $k$ is the wave number and $w$ the frequency (for simplicity, we deal only with the one-dimensional case). Taking into account Einstein's relation $E=\hbar w$, and De Broglie's one, $p=\hbar k$, he rewrote the expression for the plane waves arising in electromagnetic theory in terms of the energy and momentum, as

$$
\Phi(x, t)=\Phi_{0} e^{i(p x-E t) / \hbar} .
$$

Substituting back in (2), of course we get the relativistic energy for a photon (the quantum of the electromagnetic field)

$$
E^{2}=p^{2} c^{2}
$$

but, as previously stated, Schrödinger wanted (1) instead. What he noticed, by a simple inspection, is that this relation is precisely what results if we take as the wave equation

$$
\left(\frac{\partial^{2}}{\partial x^{2}}-\frac{1}{c^{2}} \frac{\partial^{2}}{\partial t^{2}}-\frac{m^{2} c^{2}}{\hbar^{2}}\right) \Phi(x, t)=0
$$

Schrödinger went on to study the Hidrogen atom with this equation, but soon he found that it gave an incorrect spectrum, so he discarded it as a valid quantum equation. After this, the equation was rediscovered by Pauli, Klein, Gordon and Fock, among others (Pauli called it "the equation with many fathers", see [4]), and today (3) is widely known as the Klein-Gordon equation. It is a quantum relativistic wave equation, used in the description of particles with spin 0 .

As in the case of the Schrödinger equation (which he derived later starting from the non-relativistic expression for the energy), it is instructive to explore the behavior of the solutions to the Klein-Gordon equation for some simple potentials. Perhaps the simplest is the step potential,

$$
V(x)=\left\{\begin{array}{l}
V, \text { if } x \geq 0 \\
0, \text { if } x<0
\end{array}\right.
$$

When the energy of an incident beam of particles (from the left) is $E<V$, it is well-known that for particles described by the Schrödinger equation there is some penetration in the region to the right of the barrier, expressed by the fact that there is a non vanishing transmission coefficient, but that density exponentially decays with distance.

What is surprising is that, as we shall see, when the step potential is included in the Klein-Gordon equation the barrier can become transparent at high values for $V$, and the (normalized) reflection coefficient is greater than 1 , even when the energy of the incident beam is $E<V$. This counter-intuitive behavior is called the Klein paradox. It is a common feature of quantum relativistic one-particle equations, and, indeed, it was first discussed for the Dirac equation (see [8] and [3]). 
The Klein paradox is commonly solved for the case of the Dirac equation within the framework of quantum field theory (QFT) [9]: the strong potential to the right of the barrier excites the vacuum, creating electron-positron pairs, and acts attracting the positron states, which couples with the electrons outside the barrier with the same energy. Other solutions are offered in [2] and [5]. However, there are no available experiments where this phenomenon is considered, although recent studies seem to point to bi-layer graphene as a possible setup [7]. Anyway, as stated in [1], the main point is that the paradox arises in the context of a quantum relativistic theory for one particle, so it would be desirable (to assess the selfconsistency of the theory) to know if it can be solved also without resorting to QFT. Indeed, we follow the ideas in that paper (which deals with the Dirac equation) to show that this is the case for the Klein-Gordon equation (there exists an earlier, different treatment in [13] based on the consideration of a finite non-zero width of the barrier, and [6] reviews the usual pair creation solution in this setting). As in [1], our main tool will be a suitable extension of the method of images.

The method of images is described in any textbook dealing with electrostatics as an efficient tool for studying the field created by charge distributions involving media discontinuities. Its basic idea is to introduce virtual charges inducing a field that compensates the one created by real charges, in such a way that it satisfies the prescribed boundary condition on the media discontinuities. Here, following [1], we generalize it to show that if virtual beams, instead of virtual charges, are introduced into the problem of the step potential for the Klein-Gordon equation, with suitable matching conditions, then the paradox is solved by considering the total reflection and transmission coefficients (that is, the coefficients corresponding to both the real and the virtual beams).

To make the paper relatively self-contained, we very briefly recall the basics of the method of images in Sec. 2, and reproduce in detail the computations leading to the Klein paradox in Sec. 3. Section 4 contains the solution of the problem.

\section{Preliminaries: the method of images}

Let us begin by recalling that for any linear partial differential operator of order $m$ with real-analytic coefficients on $\mathbb{R}^{n}, P(\mathbf{x}, D)$, and any non-characteristic, analytic regular submanifold $S \subset \mathbb{R}^{n}$, Holmgren's uniqueness theorem (see [10]) guarantees the uniqueness of the solution to the Cauchy problem

$$
\left\{\begin{array}{l}
P u=f \\
D^{\alpha} u=D^{\alpha} g \text { on } S
\end{array}\right.
$$

for any multi-index $0 \leq|\alpha| \leq m-1$, where $g$ is a given analytic function. This is the case, for example, of the Laplace operator $\Delta=\nabla^{2}$ or the Klein-Gordon one given in (3).
Consider now the Poisson equation, defined on a connected domain $D \subset \mathbb{R}^{n}$ with regular boundary $S=\partial D$ :

$$
\nabla^{2} u=f
$$

where $f: D \rightarrow \mathbb{R}$ is a given function, physically representing, for instance, a distribution of electrostatic sources in empty space. The fundamental solution for this problem is a distribution (in the sense of L. Schwartz, see [11] or the more physics-oriented text [12]) $F\left(\mathbf{x}-\mathbf{x}^{\prime}\right)$ such that $^{i}$

$$
\nabla^{2} F\left(\mathbf{x}-\mathbf{x}^{\prime}\right)=\delta\left(\mathbf{x}-\mathbf{x}^{\prime}\right),
$$

where $\delta$ is the Dirac (singular) distribution. Because of the property $\delta * f=f=f * \delta$, it turns out that the solution to (5) is given by convolution with the source:

$$
u=F * f .
$$

The fundamental solution is not unique, of course. For instance, the addition of any harmonic distribution $h$ (such that $\nabla^{2} h=0$ ) gives a new fundamental solution $G=F+h$. The method of images provides a judicious choice of $h$ to guarantee that prescribed boundary conditions on $S=\partial D$ are satisfied.

For the case of Dirichlet and Neumann problems, one wants that the solution $G$ to satisfy $G\left(\mathbf{x}-\mathbf{x}^{\prime}\right)=0$ for any $\mathbf{x} \in S$, and in this case it is called the Green function. Other conditions, such as asymptotic ones, can be imposed on $G$.

When the sources have support on a set $\Gamma$, given any $\mathbf{x}^{\prime} \in$ $\Gamma$ we construct the associated Green function as $G=F+h$ where

$$
\nabla^{2} h=\sum_{j=1}^{N} q_{j} \delta\left(\mathbf{x}-\mathbf{x}_{j}\right),
$$

and $q_{j}, \mathbf{x}_{j}$ are, respectively, a set of weights and positions chosen in such a way that $G=F+h$ satisfy $G\left(\mathbf{x}-\mathbf{x}^{\prime}\right)=0$ for all $\mathrm{x} \in S=\partial D$.

What we want to stress at this point is the fact that the boundary $S$ provides the conditions required to build the solution, and these conditions very often can be deduced from symmetry conditions. We will return to this point later, in Sec. 4.

The prototypical example where this method is applied is that of a harmonic function in $\mathbb{R}^{3}$ with prescribed values on the plane $z=0$. Thus, we look for a function $u(x, y, z)$ such that $\nabla^{2} u=0$ and $u(x, y, 0)=f(x, y, 0)$ for a given function $f(x, y, 0)$, which exists and is unique because the Cauchy-Kowalevskaya and Holmgren theorems. Thus, if we consider the problem of determining the field created by a single charge $q$ located at a distance $d$ of an infinite, grounded conducting plane (which plays the rôle of the surface $S$ ), we can arrange the coordinate system so the conductor coincides with the $z=0$ plane and the charge is located at $z=d$; then, the problem reduces itself to finding the location $\mathbf{x}_{1}$ of a single charge such that the total potential in the points $(x, y, 0)$ vanishes. As stated above, symmetry considerations are an essential part of the method. In the present case, the symmetry of the problem suggests to take $\mathbf{x}_{1}=(0,0,-d)$ and 
$q_{1}=-q$ in (7). The Green function becomes the sum of the fundamental solution of the Laplacian in $\mathbb{R}^{3}$ (see [12]), and the harmonic function $h$ so found:

$$
\begin{aligned}
G\left(\mathbf{x}-\mathbf{x}^{\prime}\right) & =-\frac{1}{4 \pi\left\|\mathbf{x}-\mathbf{x}^{\prime}\right\|}+h\left(\left\|\mathbf{x}-\mathbf{x}^{\prime}\right\|\right) \\
& =-\frac{1}{4 \pi\left\|\mathbf{x}-\mathbf{x}^{\prime}\right\|}+\frac{1}{4 \pi\left\|\mathbf{x}+\mathbf{x}^{\prime}\right\|},
\end{aligned}
$$

and the uniqueness property assures that the solution for the potential in the region $z>0$ is the well known expression

$$
\begin{aligned}
V(x, y, z) & =\frac{q}{4 \pi}\left(\frac{1}{\sqrt{x^{2}+y^{2}+(z-d)^{2}}}\right. \\
& \left.+\frac{1}{\sqrt{x^{2}+y^{2}+(z+d)^{2}}}\right) .
\end{aligned}
$$

\section{The Klein paradox}

Let us simplify the notation by taking natural units $(\hbar=1=c)$, so the Klein-Gordon equation in three spatial dimension becomes

$$
\left(\frac{\partial}{\partial t^{2}}-\Delta+m^{2}\right) \Phi(\mathbf{x}, t)=0,
$$

or, in terms of the operators $\partial_{\mu}=(\partial / \partial t, \nabla)$ and $\partial^{\mu}=$ $\eta^{\mu \nu} \partial_{\nu}=(\partial / \partial t,-\nabla)\left(\right.$ where $\eta_{\mu \nu}=\operatorname{diag}(1,-1,-1,-1)$ is Minkowski's metric),

$$
\left(\partial_{\mu} \partial^{\mu}+m^{2}\right) \Phi(x, t)=0 .
$$

The relativistic probability current for the Klein-Gordon equation is defined as the 4 -vector

$$
j^{\mu}=-\frac{1}{2 m i}\left(\Phi^{*} \partial^{\mu} \Phi-\Phi \partial^{\mu} \Phi^{*}\right) .
$$

It is readily seen that $\partial_{\mu} j^{\mu}=0$, that is, the probability current is conserved. The associated current density is given, as usual, by the temporal component:

$$
\begin{aligned}
\rho=j^{0} & =-\frac{1}{2 m i}\left(\Phi^{*} \partial^{0} \Phi-\Phi \partial^{0} \Phi^{*}\right) \\
& =-\frac{1}{2 m i}\left(\Phi^{*} \partial_{t} \Phi-\Phi \partial_{t} \Phi^{*}\right),
\end{aligned}
$$

so, from the conservation of $j^{\mu}$ we get the continuity equation

$$
\partial_{\mu} j^{\mu}=\frac{\partial \rho}{\partial t}+\nabla \mathbf{j}=0
$$

where $j^{\mu}=(\rho, \mathbf{j})$. Notice that, for a free plane wave solution of definite $3-$ momentum $\mathbf{p}, \Phi(\mathbf{x}, t)=e^{-i(E t-\mathbf{p} \cdot \mathbf{x})}$, the density is given by

$$
\rho(\mathbf{x}, t)=\frac{E}{m},
$$

and the fact that we are dealing with relativistic particles, where the energy and momentum are related through (1), implies that $E$ can take any of the values $E= \pm \sqrt{\|\mathbf{p}\|^{2}+m^{2}}$, so $\rho$ is not positive-definite and cannot be interpreted as a probability density (that is the reason for calling it a current density instead). This appearance of negative energies lies at the heart of the Klein paradox, as we will see in what follows.

When including interaction with an electromagnetic field, use must be made of the minimal coupling, replacing $p_{\mu}$ by $p_{\mu}-A_{\mu}$ (natural units), where $A_{\mu}=(V, \mathbf{A})$ is the electromagnetic potential. In this case, it is easy to see that the density associated to the the conserved current is

$$
\rho=-\frac{1}{2 m i}\left(\Phi^{*} \partial_{t} \Phi-\Phi \partial_{t} \Phi^{*}\right)-\frac{1}{m} V \Phi^{*} \Phi,
$$

instead of (8)

Consider now a beam of particles in one spatial dimension, of positive unit charge, described by the Klein-Gordon equation in the presence of a step (electric) potential (4). If the particles fall on the barrier from the left, with an energy $E$ satisfying $0<E<V$, by defining the effective momenta

$$
k_{1}=+\sqrt{E^{2}-m^{2}}, \quad k_{2}=+\sqrt{(V-E)^{2}-m^{2}},
$$

it is readily found that the solution with positive energies is given by

$$
\Phi(x, t)=\left\{\begin{array}{l}
e^{-i E t} u_{I}(x), \quad x<0 \\
e^{-i(V-E) t} u_{I I}(x), \quad x \geq 0,
\end{array}\right.
$$

with the stationary functions

$$
\left\{\begin{array}{l}
u_{I}=e^{i k_{1} x}+A e^{-i k_{1} x}, \quad x<0 \\
u_{I I}=C e^{i k_{2} x}+B e^{-i k_{2} x}, \quad x \geq 0,
\end{array}\right.
$$

and where we have normalized the incident beam, so the coefficient of the fraction that propagates to the right in the region $x<0$ is 1 . Notice that this solution is a superposition of beams propagating to the right and to the left in both regions, $x<0$ (region I), and $x \geq 0$ (region II). Our identification of the incident beam is based upon the fact that the current density in region I is given by (8),

$$
\begin{aligned}
\rho_{I} & =-\frac{1}{2 m i}\left(\Phi^{*} \partial_{t} \Phi-\Phi \partial_{t} \Phi^{*}\right) \\
& =\frac{i}{2 m}\left(-2 i E u_{I}^{*} u_{I}\right)=\frac{E}{m}\left|u_{I}\right|^{2},
\end{aligned}
$$

so it has the same sign as the incident charge, while in the region II it has the opposite sign, because $V>E$ in (9),

$$
\rho_{I I}=\frac{i}{2 m}\left(-2 i E u_{I I}^{*} u_{I I}\right)-\frac{V}{m} u_{I I}^{*} u_{I I}=\frac{E-V}{m}\left|u_{I I}\right|^{2} .
$$

Thus, in the expression for $u_{I I}$ we find that the term $C e^{i k_{2} x}$ has an associated current running from left to right, with a negative current density $\rho_{I I}=|C|^{2}(E-V) / m$; equivalently, we could say that it describes a current of positive charges running from right to left. Analogously, the term $B e^{-i k_{2} x}$ has an associated current running from right to left with negative current density, so it can be interpreted as a positive 
current from left to right. As the only charges present are positive, and there are no sources to the right of the barrier, we must take $C=0$ in (12), arriving at the proposed solution

$$
\left\{\begin{array}{l}
u_{I}=e^{i k_{1} x}+A e^{-i k_{1} x}, \quad x<0 \\
u_{I I}=B e^{-i k_{2} x}, \quad x \geq 0
\end{array}\right.
$$

As a technical remark, let us note that a straightforward computation with the wavefunction (11) (where the stationary solutions are given by (13)) gives

$$
\begin{aligned}
& \left(\partial_{\mu} \partial^{\mu}+m^{2}\right) \Phi(x, t) \\
& \quad=\left\{\begin{array}{l}
\left(m^{2}-E^{2}+k_{1}^{2}\right) \Phi(x, t), \quad x<0 \\
\left(m^{2}-(V-E)^{2}+k_{2}^{2}\right) \Phi(x, t), \quad x \geq 0,
\end{array}\right.
\end{aligned}
$$

so, in order to be a solution of the Klein-Gordon equation, $\Phi(x, t)$ must have its support contained in the hyperboloids $m^{2}-E^{2}+k_{1}^{2}=0$, for $x<0$, and $m^{2}-(V-E)^{2}+k_{1}^{2}=0$, for $x \geq 0$. These on-shell conditions are automatically satisfied because of the definitions (10) of the effective momenta.

In this way, we have identified the incident, reflected and transmitted beams, $u_{i}, u_{r}$, and $u_{t}$, respectively given as $u_{i}=e^{i k_{1} x}, u_{r}=A e^{-i k_{1} x}$, and $u_{t}=B e^{-i k_{2} x}$. Now, we can compute the corresponding incident, reflected, and transmitted currents:

$$
\begin{aligned}
& j_{i}=\frac{1}{2 m i}\left(u_{i}^{*} \frac{\partial}{\partial x} u_{i}-u_{i} \frac{\partial}{\partial x} u_{i}^{*}\right)=\frac{k_{1}}{m}, \\
& j_{r}=\frac{1}{2 m i}\left(u_{r}^{*} \frac{\partial}{\partial x} u_{r}-u_{r} \frac{\partial}{\partial x} u_{r}^{*}\right)=-\frac{|A|^{2} k_{1}}{m}, \\
& j_{t}=\frac{1}{2 m i}\left(u_{t}^{*} \frac{\partial}{\partial x} u_{t}-u_{t} \frac{\partial}{\partial x} u_{t}^{*}\right)=-\frac{|B|^{2} k_{2}}{m} .
\end{aligned}
$$

Then, we get for the reflection coefficient the expression

$$
R=\left|\frac{j_{r}}{j_{i}}\right|=|A|^{2},
$$

while the transmission coefficient is

$$
T=\frac{j_{t}}{j_{i}}=-\frac{k_{2}}{k_{1}}|B|^{2} .
$$

Imposing the continuity conditions at the barrier for the wave function and its derivative, $u_{I}(0)=u_{I I}(0)$, and $u_{I}^{\prime}(0)=$ $u_{I I}^{\prime}(0)$, we get a system of equations for $A, B$ whose solution is

$$
A=\frac{k_{1}+k_{2}}{k_{1}-k_{2}}, \quad B=\frac{2 k_{1}}{k_{1}-k_{2}},
$$

so we can write, in terms of the effective momenta,

$$
R=\left(\frac{k_{1}+k_{2}}{k_{1}-k_{2}}\right)^{2}, \quad T=-\frac{k_{2}}{k_{1}}\left(\frac{2 k_{1}}{k_{1}-k_{2}}\right)^{2} .
$$

Thus, the reflection and transmission coefficients make a pretense of respecting the conservation of matter, as a straightforward computations shows that

$$
R+T=1
$$

However, a look at (14) reveals that $T$ is negative, an already shocking fact, but even more so since it implies $R>1$, meaning that there are more reflected than incident particles. This is the Klein paradox.

\section{Virtual beams and resolution of the para- dox}

We will exploit the similarity between the geometric setup of the Klein-Gordon paradox and that of the method of images. In both cases we have a surface of discontinuity which determines some conditions to be satisfied by the solution to the problem although, of course, there is a fundamental difference: The method of images is applied to a static charge configuration, while in the Klein-Gordon case we have density currents; but let us focus on the formal similarities as a means to obtain an ansatz for the structure of the solution.

Following the analogy with the method of images, we look for a way to compensate the excess in the reflection coefficient $R>1$, found in the preceding section, by considering it as a measure of the flux of particles in a beam through the discontinuity surface determined by the barrier. Thus, we will construct virtual beams out from symmetry considerations in such a way that the net flux through the barrier, from left to right, gives $R<1$. Similarly, the net transmission coefficient must be $T<1$ while their sum satisfies $R+T=1$. As it is the case with the virtual image introduced in the example of Sec. 2 , these virtual beams are not observable, so they can be safely incorporated to the solution.

Now, recall that the Green solution given by the method of images has the structure $G=F+h$, where $F$ is a fundamental solution and $h$ is chosen so $G$ satisfies the prescribed boundary conditions. Let us choose as the analog of $F$ the wavefunction $\Phi(x, t)$ given by (11) with (13). Due to the symmetry of the problem, as the function $h$ we choose the wavefunction describing a beam of particles incident on the barrier from right to left, whose stationary part is given by

$$
w(x)=\left\{\begin{array}{l}
w_{I}=e^{i k_{2} x}+C e^{-i k_{2} x}, \quad x \geq 0 \\
w_{I I}=D e^{-i k_{1} x}, \quad x<0 .
\end{array}\right.
$$

It is straightforward to compute, for the function $\Psi(x, t)=$ $e^{-i E t} w_{I}$ on $x<0$, and $\Psi(x, t)=e^{-i(V-E) t} w_{I I}$ on $x \geq 0$, that

$$
\begin{aligned}
\left(\partial_{\mu} \partial^{\mu}\right. & \left.+m^{2}\right) \Psi(x, t) \\
& =\left\{\begin{array}{c}
\left(m^{2}-E^{2}+k_{1}^{2}\right) \Psi(x, t), \quad x<0 \\
\left(m^{2}-(V-E)^{2}+k_{2}^{2}\right) \Psi(x, t), \quad x \geq 0,
\end{array}\right.
\end{aligned}
$$

so it is supported on the same subset as $\Phi(x, t)$. Thus, onshell we have two solutions to the Klein-Gordon equation and by linearity we can form their superposition. Actually, we only need to work with the stationary solutions, so we will forget about the time dependence in what follows. 
Let us compute the reflection and transmission coefficients for the solution $w$. A reasoning completely analogous to that developed in the preceding section leads to the incident, reflected and transmitted beams $w_{i}, w_{r}$, and $w_{t}$ given by $w_{i}=e^{i k_{2} x}, w_{r}=C e^{-i k_{2} x}$, and $w_{t}=D e^{-i k_{1} x}$, respectively. Then, we can compute the corresponding currents (where we have added a superscript to make clear the solution used),

$$
\begin{aligned}
& j_{i}^{w}=\frac{1}{2 m i}\left(w_{i}^{*} \frac{\partial}{\partial x} w_{i}-w_{i} \frac{\partial}{\partial x} w_{i}^{*}\right)=\frac{k_{2}}{m} \\
& j_{r}^{w}=\frac{1}{2 m i}\left(w_{r}^{*} \frac{\partial}{\partial x} w_{r}-w_{r} \frac{\partial}{\partial x} w_{r}^{*}\right)=-\frac{|C|^{2} k_{2}}{m}, \\
& j_{t}^{w}=\frac{1}{2 m i}\left(w_{t}^{*} \frac{\partial}{\partial x} w_{t}-w_{t} \frac{\partial}{\partial x} w_{t}^{*}\right)=-\frac{|D|^{2} k_{1}}{m} .
\end{aligned}
$$

For this virtual beam, the expressions of the reflection and transmission coefficients are

$$
R^{w}=\left|\frac{j_{r}^{w}}{j_{i}^{w}}\right|=|C|^{2}, \quad T^{w}=\frac{j_{t}^{w}}{j_{i}^{w}}=-\frac{k_{1}}{k_{2}}|D|^{2} .
$$

A look at these formulas reveals a symmetric behavior with respect to the wavefunction that represents the beam running from left to right. Something more can be said if we consider the continuity conditions for $w(x)$ and $w^{\prime}(x)$ at the barrier. Indeed, these conditions lead to a system of equations for $C$, $D$ with solution

$$
C=\frac{k_{1}+k_{2}}{k_{2}-k_{1}}, \quad D=\frac{2 k_{2}}{k_{2}-k_{1}},
$$

leading to the following expressions for $R^{w}, T^{w}$, in terms of the effective momenta,

$$
R^{w}=\left(\frac{k_{1}+k_{2}}{k_{2}-k_{1}}\right)^{2}, \quad T^{w}=-\frac{k_{1}}{k_{2}} \frac{4 k_{2}^{2}}{\left(k_{2}-k_{1}\right)^{2}} .
$$

It is readily seen that, again, $R^{w}>1$ and $T^{w}<0$. Moreover, we have the fake conservation relation

$$
R^{w}+T^{w}=1 .
$$

However, the superposition $u(x)+w(x)$ solves all the problems. It has associated a global reflection coefficient obtained by considering the fraction of particles (whatever their charges) that after the dispersion lie at the left of the barrier. This fraction come from two sources. On the one hand, we have the reflected particles that were incident form the left. On the other hand, there are the transmitted virtual particles that were incident from the right. This is analogous as the computation of the potential on the boundary surface in the method of images, where we superimpose the potential of the real and virtual charges.

Therefore, the global reflection coefficient $R_{G}$ can be computed by adding the reflection coefficient for the original beam, $R^{u}$ in (14) (putting the $u$ superscript for clarity), and the transmission coefficient for the virtual beam, $T^{w}$ in (15). We then obtain, after some simple algebraic manipulations,

$$
R_{G}=R^{u}+T^{w}=\left(\frac{k_{1}+k_{2}}{k_{1}-k_{2}}\right)^{2}-\frac{k_{1}}{k_{2}} \frac{4 k_{2}^{2}}{\left(k_{2}-k_{1}\right)^{2}}=1 .
$$

This result can be interpreted by saying that the superposition $u(x)+w(x)$ leads to a total global reflection of the particles to the left, what is to be expected since the incident energy is $E<V$, thus solving the paradox.

\section{Conclusions}

The Klein paradox appears for any relativistic quantum wave equation when studying the dispersion by a discontinuous potential. Following the treatment in [1] for the Dirac equation, we have shown that in the case of the Klein-Gordon equation the excess in the reflection coefficient can be explained by the introduction of virtual beams, analog to the introduction of virtual charges in the well-known method of images of electrostatics. A detailed mathematical analysis of this method allows a reasonable understanding of the paradox within the context of the quantum relativistic theory of one particle, without resorting to quantum field theoretic ideas.

\section{Acknowledgements}

A. Molgado thanks the financial support from CONACyTMéxico under project number CB-2014-243433. José A. Vallejo was partially supported by a project CONACyTMéxico CB-2012-179115. $i$. Note that, for a general differential operator, $F$ will not be a regular distribution, much less will have a functional dependence on $\mathbf{x}, \mathbf{x}^{\prime} \in \mathbb{R}^{n}$ through the difference $\mathbf{x}-\mathbf{x}^{\prime}$. In this case, however, this happens because the Laplace operator has constant coefficients, so translational invariance applies.

1. A. D. Alhaidari, Physica Scripta 83 (2011) 025001.

2. S. Danko-Bosanac, J. Phys. A: Math. Theor. 40 (2007) 89919001.

3. N. Dombey and A. Calogeracos, Phys. Rep. 315 (1999) 41-58.
4. C.P. Enz: No Time to be Brief: A Scientific Biography of Wolfgang Pauli. (Oxford UP, 2002).

5. A. Hansen and F. Ranvdal, Physica Scripta 23 (2008) 10361042.

6. B.R. Holstein, Am. J. Phys. 66 (1998) 507-512.

7. M.I. Katsnelson, K.S. Novoselov and A.K. Geim, Nature Physics 2 (2006) 620-625.

8. O. Klein, Z. Phys. 53 (1929) 157-165. 
9. P. Krekora, Q. Su and R. Grobe, Phys. Rev. Lett. 92 (2004) 040406.

10. J. Rauch: Partial Differential Equations. (Springer Verlag, New York, 1991).
11. L. Schwarz: Théorie des distributions. (Hermann, Paris, 1950).

12. T. Schücker: Distributions, Fourier transforms and some of their applications to Physics. (World Scientific, 1991).

13. R.G. Winter, Am. J. of Phys. 27 (1959) 355-358. 